\title{
MULTIPLE CRITERIA OPTIMISATION APPROACH TO DERIVING PRIORITIES IN THE ANALYTIC HIERARCHY PROCESS
}

\author{
Ludmil Mikhailov \\ Department of Computation, UMIST \\ Sackville Street, PO Box 88, Manchester M60 1QD, U.K. \\ E-mail: L.Mikhailov@co.umist.ac.uk
}

Keywords: multiple criteria analysis, multiple objective programming, AHP, prioritisation methods

Summary: This paper is concerned with the problem of deriving priority vectors from pairwise comparison judgements, in the framework of the Analytic Hierarchy Process. With the exception of the traditional Eigenvalue method, all other prioritisation methods are based on minimisation of an objective function. However, a single objective function cannot encompass and satisfy all requirements about the quality of solutions. The multiple criteria character of the requirements necessitates a multiple objective approach to prioritisation, rather than optimisation of a single objective function. The paper proposes a new multi-criteria prioritisation approach, minimising the Euclidean norm and the number of rank violations, which measure the most important properties of the solutions. The numerical results show that the proposed two-objective method outperforms the known prioritisation methods, with respect to accuracy and rank preservation requirements.

\section{Introduction}

The assessment of weights of criteria and scores of alternatives is one of the most important tasks in the multicriteria decision-making. In the Analytical Hierarchy Process (AHP), proposed by Saaty [18], the values of weights and scores are assessed indirectly from comparison judgements. The elicitation process for both weights and scores is the same, so they are often called priorities.

The pairwise comparison process in the AHP assumes that the decision-maker can compare any two elements at a given hierarchical level and to provide a numerical value of the ratio of their importance. Comparing any two elements $E_{i}$ and $E_{j}$, the decision-maker assigns a value $a_{i j}$, which represents a judgement concerning the relative importance of preference of the decision element $E_{i}$ over $E_{j}$. If $E_{i}$ is preferred to $E_{j}$ then $a_{i j}>1$, otherwise $0<a_{i j} \leq 1$.

A full set of judgements for a level with $n$ elements requires $n(n-1) / 2$ comparisons. In order to derive a priority vector $w=\left(w_{1}, w_{2}, \ldots, w_{n}\right)^{T}$ from a given set of judgements, Saaty [18] constructs a positive reciprocal matrix $A=\left\{a_{i j}\right\} \in \mathfrak{R}^{n x n}$. The Eigenvector prioritisation method (EV) [18], [19] is based on some properties of the pairwise comparison matrices. Using the Perron-Frobenius theorem, Saaty proves that the principal right eigenvector of $A$ can be used as a priority vector.

With the exception of the traditional EV method, all other methods for deriving priorities in the AHP are based on some optimisation approach. They introduce an objective function, which measures the degree of approximation or the distance between an "ideal" solution and the actual one. Thus the problem of priority derivation is formulated as an optimisation task of minimising the objective function, subject to normalisation and some additional constraints. 
The Direct Least Squares (DLS) method [5], [11], [17] is based on the assumption that the errors between the initial judgements $a_{i j}$ and solution ratios $w_{i} / w_{j}$ should be minimised, so it uses the Euclidean distance metric as an objective function. The objective function, used in the Weighted Least Squares (WLS) method [3], [5] is also a $L^{2}$ norm, similar to that of the DLS. The WLS transforms the solution to the problem to a system of linear equations that can easily be solved. It is shown in [3] that the WLS, unlike the DLS, provides a unique solution.

The Logarithmic Least Squares (LLS) method of Crawford and Williams [7] makes use of the multiplicative properties of the pairwise comparison matrices and applies an optimisation procedure, minimising the logarithm of the squared Euclidean distance. This method gives an explicit solution, which is rather simple and convenient from a computational point of view.

Some authors formulate the prioritisation task as a goal programming (GP) problem by introducing additional deviation variables [4], [8]. The objective function to be minimised is expressed as a sum of these deviation variables. For example, the logarithmic goal programming method proposed by Bryson [4] minimises a linear logarithmic function of deviation variables subject to a number of linear constraints.

The Fuzzy Preference Programming (FPP) method, proposed by the author of this paper [14] introduces fuzzy sets that measure the degree of approximation of the fuzzy equalities $w_{i}-a_{i j} w_{j} \cong 0$. The prioritisation problem is formulated as a fuzzy linear programming model that maximises the overall degree of satisfaction with a given priority vector.

All prioritisation methods mentioned above try to find a solution which best satisfy the initial judgements, but the notion of 'best' is defined in different ways by their objective functions. Obviously, the properties of the prioritisation methods and their solutions are closely related to those of their metrics. So, the natural question is which metric has to be preferred by the decision-maker for solving a specific prioritisation problem.

There is an ongoing discussion in the literature about the advantages and limitations of the various prioritisation methods. The researchers recognise the multi-criteria nature of the evaluation problem and explore a number of properties, applying various evaluation criteria. The most important properties of the prioritisation methods, identified in these comparison studies are the accuracy of solutions, rank preservation and invariance to transposition.

Usually, the accuracy of prioritisation methods is measured by the Euclidean distance, which is the metric of the DLS method. Many researchers have applied the Euclidean distance as a criterion, comparing different prioritisation methods [5], [7], [8], [10], [15], [19], [22], [23]. Some authors evaluate additional properties of these methods, as rank preservation and invariance to transposition, using additional criteria [2], [3], [9], [10], [15], [19], [11], [23].

The main conclusion from most comparison studies is that there is no prioritisation method that is superior to the others with respect to all evaluation criteria. All methods have their advantages and drawbacks and the choice of the prioritisation method should be dictated by the objective of the analysis.

Despite the multi-criteria nature of the requirements, regarding the properties of their solutions, all extremal prioritisation methods optimise a single objective function. However, as the comparison studies show, a single objective function cannot encompass and satisfy all requirements. The multiple requirements about the quality of solutions require development of multi-criteria prioritisation methods, which could provide better results, than the existing single objective optimisation methods.

The main objective of this paper is to propose a new multi-criteria optimisation approach to prioritisation. A two-objective prioritisation (TOP) problem is formulated as an optimisation task for minimisation of the Euclidian norm and the number of rank violations. A method and algorithm for solving the TOP problem is proposed, and conditions for obtaining a Pareto optimal set of solutions are derived. Finally, a 
numerical example is given, where the solutions to the TOP problem are compared to those, obtained by some other prioritisation methods.

\section{Multiple-objective prioritisation problem}

\subsection{Optimisation criteria}

Let $S=\left\{a_{i j} \mid j>i\right\}$ be a set of pairwise comparison judgements. The feasible set $Q$ is defined as the set of all priority vectors $w=\left(w_{1}, \ldots, w_{n}\right)^{T}$, which satisfy the normalisation and non-negativity constraints:

$$
Q=\left\{\left(w_{1}, \ldots, w_{n}\right) \mid w_{i}>0, \sum_{i=1}^{n} w_{i}=1\right\}
$$

The accuracy of the each priority vector $w \in Q$, approximately satisfying the comparison judgements can be measured by the Total deviation criterion

$$
T(w)=\sum_{i=1}^{n-1} \sum_{j=i+1}^{n}\left(a_{i j}-\frac{w_{i}}{w_{j}}\right)^{2}
$$

This criterion is equivalent to the squared Euclidean distance for the upper triangular part of Saaty's reciprocal matrix $A$.

The rank preservation properties of the solutions can be measured by the Minimum violations criterion [1], [10]:

$$
M V=\sum_{i=1}^{n-1} \sum_{j=i+1}^{n} v_{i j}
$$

where

$$
v_{i j}=\left\{\begin{array}{cc}
1, & \text { if } w_{i}>w_{j} \text { and } a_{i j}<1, \text { or } w_{i}<w_{j} \text { and } a_{i j}>1, \\
1 / 2, & \text { if } w_{i}=w_{j} \text { and } a_{i j} \neq 1, \text { or } w_{i} \neq w_{j} \text { and } a_{i j}=1, \\
0, & \text { otherwise. }
\end{array}\right.
$$

The Minimum violation criterion (3) can be represented in the following compact form:

$$
V(w)=\frac{1}{2} \sum_{i=1}^{n-1} \sum_{j=i+1}^{n}\left|\operatorname{signum}\left(a_{i j}-1\right)-\operatorname{signum}\left(\frac{w_{j}}{w_{j}}-1\right)\right|,
$$

where the signum function is defined as:

$$
\operatorname{signum}(b)= \begin{cases}1, & \text { if } b>0 \\ 0, & \text { if } b=0 \\ -1, & \text { if } b<0\end{cases}
$$

\subsection{Statement of the optimisation problem}

The two-objective prioritisation (TOP) problem is to find a feasible priority vector that 'simultaneously' minimises the Total deviation and the Number of violations:

minimise $T(w)$ and $V(w)$ 
subject to $w \in Q$,

where $T: R^{n} \rightarrow R^{1}$ and $V: R^{n} \rightarrow R^{1}$ are real-valued objective functions, defined by (2) and (4) correspondingly.

Each feasible vector $w \in Q$ determines a unique value of the objective function vector $y=(T(w), V(w))$. Therefore, the feasible set $Q$ in the space of decision variables can be transformed into a payoff set $Y$ in the two-dimensional objective space:

$$
Y=\left\{y \mid y=\left(y_{1}, y_{2}\right) \in \mathfrak{R}^{2} \text { such that } y_{1}=T(w) \text { and } y_{2}=V(w), w \in Q\right\} .
$$

The payoff set Y represents a feasible region of the admissible values of $T(w)$ and $V(w)$, and can be considered as the image of the feasible set $Q$ in the objective space. The payoff set $Y$ of the TOP problem consists in parallel line segments, as the function $V(w)$ takes non-negative discrete values in some range, and the function $T(w)$ is bounded.

\subsection{Solutions to the TOP problem}

Definition 1. A priority vector $w^{*} \in Q$ is said to be an optimal (superior) solution to the TOP problem (5) if it attains the minimum values of both criteria simultaneously.

Mathematically, $w^{*}$ is an optimal solution to the problem if and only if $T\left(w^{*}\right) \leq T(w)$ and $V\left(w^{*}\right) \leq V(w)$ for all $w \in Q$. Optimal solutions to the TOP problem exist only if the set of pairwise comparison judgements $S$ is totally or strongly transitive. If the judgements are weakly transitive or non-transitive, the TOP, as most multi-objective problems, has no optimal solution.

Let $\mathrm{T}^{*}$ and $\mathrm{V}^{*}$ be global minima of the single objective problems:

$$
\begin{aligned}
& T^{*}=\min \{T(w) \mid w \in Q\}, \\
& V^{*}=\min \{V(w) \mid w \in Q\} .
\end{aligned}
$$

Then $y^{*}=\left(T^{*}, V^{*}\right)$ is called an ideal point (or utopia point) of the problem (5). If $y^{*}$ is feasible, $y^{*} \in Y$, the solution $w^{*}=(T, V)^{-1}\left(y^{*}\right)$ simultaneously minimises both objective functions.

If the set of pairwise comparison judgements $S$ is weakly transitive on non-transitive, the objectives (2) and (4) are conflicting and cannot be optimised simultaneously, so an optimal solution does not exist. Some compromise solutions, however, could be found.

Definition 2. A priority vector $w^{o} \in Q$ is said to be a Pareto optimal (or strongly non-dominated) solution if there is no $w \in Q$ such that $T(w) \leq T\left(w^{0}\right)$ and $V(w) \leq V\left(w^{0}\right)$, with a strict inequality for at least one of these conditions.

The above definition implies that if $w^{o}$ is a Pareto optimal solution to the TOP problem (5), the value of $T\left(w^{0}\right)$ cannot be decreased without causing a simultaneous increase in the Number of violations, and wise versa.

Definition 3. A priority vector $w^{o} \in Q$ is said to be a weak Pareto optimal (or weakly non-dominated) solution if there is no $w \in Q$ such that $T(w)<T\left(w^{0}\right)$ and $V(w)<V\left(w^{0}\right)$. 
It is obvious that Pareto optimality implies weak Pareto optimality, i.e. if $w^{o}$ is strongly non-dominated, then it is also weakly non-dominated. If $w^{o}$ is a weak Pareto optimal solution, then it is not possible to decrease simultaneously the values of both objective functions $T\left(w^{0}\right)$ and $V\left(w^{0}\right)$ by choosing another feasible solution.

Generally, the Pareto optimal solutions to the TOP problem are not unique, especially in weak transitive and non-transitive cases. We will call the set $P$ of all Pareto optimal solutions a Pareto optimal set, $P=\left\{w^{0} \mid w^{0}\right.$ is a Pareto optimal solution $\}$.

The objective vectors, corresponding to the Pareto optimal solutions $y=\left(T\left(w^{0}\right), V\left(w^{0}\right)\right)$ form the Pareto optimal front $C=\{y=(T(w), V(w)) \mid w \in P\}$. It is easy to be shown that $C$ is on the boundary of the payoff set $Y$. Indeed, for any point $y=(T, V)$ in the interior of $Y$, reduction of $T$ could be achieved by moving along the line $V=$ const., towards the point $T=0$, until the boundary of $Y$ is reached.

Since $Y$ is non-connected set, $C$ is a discrete curve in $\mathfrak{R}^{2}$, consisting of isolated points. It is also called a trade-off function, since it shows how much the value of $T$ must change to stay in $C$ when the value of $V$ changes.

Necessary conditions for a point to be in the Pareto optimal front $C$ are developed for convex and continuous objective functions [20], [21], but because of the non-continuous nature of $V(w)$ they cannot be applied directly. But we can find solutions to the TOP problem by applying an appropriate solution method.

\section{Solution to the TOP problem}

\subsection{Solution methods}

There are a number of MCDM methods that can be applied for finding Pareto optimal solutions to the two-objective prioritisation problem (5). For example, the classical weighted sum method [21] requires aggregation of the objectives into a single, parameterised objective function. Then the TOP problem can be formulated as

$$
\begin{aligned}
& \text { Minimise } J=k_{1} T(w)+k_{2} V(w) \\
& \text { subject to } w \in Q
\end{aligned}
$$

where $k_{1}>0$ and $k_{2}>0$ are weighting coefficients, which determine the relative importance of the objectives.

The main disadvantage of this method, however, is that it cannot generate all Pareto optimal solutions, if the payoff set $Y$ is non-convex. The limitations of the Weighted sum method could be overcome by the $\varepsilon$ constraint approach [13], [21], which requires specification of the maximum allowable level $\varepsilon$ of Number of violations $V(w)$, and solves the following problem:

$$
\begin{aligned}
& \text { Minimise } T(w) \\
& \text { subject to } V(w) \leq \varepsilon, \quad w \in Q
\end{aligned}
$$

The Proper Equality Constraints (PEC) method, proposed by Lin [12] is similar to the $\varepsilon$-constraint approach, but it uses only equality constraints. Lin proves that this method can also find the whole set of Pareto optimal solutions. 
Since the objective function (4) can take only a finite number of discrete values, equality constraints will be used for solving the TOP problem.

\subsection{Constrained Least Squares optimisation problem}

Let $Q_{\alpha}$ be a subset of the feasible area $Q_{\alpha} \subset Q$, such that $Q_{\alpha}=\{w \in Q \mid V(w)=\alpha\}$, where $\alpha$ is a real number, $\alpha=0.5 k, \mathrm{k}=0,1,2, \ldots \mathrm{K}$. We can denote by $\mathrm{A}$ the set of all $\alpha$ in $\mathfrak{R}$ for which the corresponding subset $Q_{\alpha}$ is nonempty. Since $\alpha$ can take only discrete values, the set $\mathrm{A}$ is also a discrete set in $\mathfrak{R}$. In order to determine the set $\mathrm{A}$, we have to find the range of $\alpha$ in $\mathfrak{R}$ such that the corresponding subsets $Q_{\alpha}$ are nonempty.

As $Q_{\alpha}$ is nonempty and closed, and $T(w)$ is bounded from below, for any $\alpha$ in $\mathrm{A}$ there exists an $\alpha$ optimal solution $\hat{w}(\alpha)$ to the following Constrained Least Squares (CLS) problem:

$$
\begin{aligned}
& \text { minimise } T(w) \\
& \text { subject to } \\
& w \in Q, V(w)=\alpha .
\end{aligned}
$$

The minimum of the objective function $\psi(\alpha)=\min \left\{T(w) \mid w \in Q_{\alpha}\right\}$ is given by $\psi(\alpha)=T(\hat{w}(\alpha))$. Since the function $\psi(\alpha)$ is defined on $\mathrm{A}$, which is a discrete set, this function is also discontinuous.

Each pair $(\alpha, \psi(\alpha)), \alpha \in \mathrm{A}$, represents a minimising vector in the payoff set $\mathrm{Y}$, attainable by the $\alpha$ optimal solution $\hat{w}(\alpha)$. The set of all pairs $L=\{(\alpha, \psi(\alpha)) \mid \alpha \in \mathrm{A}\}$ forms a discrete front in Y. Generally not all $\alpha$-optimal solutions are Pareto optimal, so $L$ is not a Pareto optimal front.

Theorem 1. For a given scalar $\alpha^{0} \in \mathrm{A}$, the solution to (10) $w^{0}=\hat{w}\left(\alpha^{0}\right)$ is Pareto optimal, if $\psi(\alpha)>\psi\left(\alpha^{0}\right)$ for any $\alpha$ in A such that $\alpha<\alpha^{0}$.

Proof: Assume that $w^{0}=\hat{w}\left(\alpha^{0}\right)$ is not Pareto optimal solution. Then, for some $\alpha \neq \alpha^{0}$ there should exist a Pareto optimal vector $w \in Q_{\alpha}$. From the Pareto optimality conditions it follows that $\alpha=V(w) \leq V\left(w^{0}\right)=\alpha^{0}$, but since $\alpha \neq \alpha^{0}$, a strict equality $\alpha<\alpha^{0}$ must hold.

As $w$ is Pareto optimal, it should be also an $\alpha$-optimal solution such that $\min \left\{T(w) \mid w \in Q_{\alpha}\right\}=\psi(\alpha)$. Then from the Pareto optimality conditions we should have $\psi(\alpha)=T(w) \leq T\left(w^{0}\right)=\psi\left(\alpha^{0}\right)$, so the $\alpha^{0}$ optimal solution satisfies the condition $\psi(\alpha) \leq \psi\left(\alpha^{0}\right)$. Therefore, if $w^{0}$ is a Pareto optimal solution, then the conditions of Theorem 1 hold. I

Using this theorem we can determine the entire set of all Pareto optimal solutions from the set $L$ of all $\alpha$ optimal solutions to the CLS problem (10) by discarding the vectors, which do not satisfy the conditions of this theorem. In order to provide a simple test for Pareto optimality, we can use the left-increasing property of the function $\psi(\alpha)$.

Definition 4. Let $\alpha^{0}$ is a point in A. Then the function $\psi(\alpha)$ is left-increasing on $\mathrm{A}$ if $\psi(\alpha)>\psi\left(\alpha^{o}\right)$ for any $\alpha$ such that $\alpha<\alpha^{0}$. 
Corollary 1. For a given point $\alpha^{0}$ in $\mathrm{A}$, the CLS solution $w^{0}=\hat{w}\left(\alpha^{0}\right)$ is Pareto optimal, if the function $\psi(\alpha)$ is left-increasing from $\alpha^{0}$.

Corollary 2. For a given point $\alpha^{0}$ in A, the CLS solution $w^{0}=\hat{w}\left(\alpha^{0}\right)$ is Pareto optimal, if the ratio

$$
\nabla \psi(\alpha)=\frac{\psi\left(\alpha^{0}\right)-\psi(\alpha)}{\alpha^{0}-\alpha}
$$

is negative for any minimising vector $(\alpha, \psi(\alpha))$ in the objective space, such that $\alpha<\alpha^{0}$.

Unlike the conditions for optimality of continuous functions, which require differentiation and might appear to be a formidable task, the above condition (11) provides a simple practical test for checking the solutions of the CLS problem for Pareto optimality, which does not need evaluating the function $\psi(\alpha)$ over A. It follows that we can obtain the set of all Pareto optimal solutions by determining the set A, solving a number of CLS problems for each $\alpha$ in $\mathrm{A}$ and then discarding all solutions, which do not satisfy (11).

However, some elements $\alpha$ in A that cannot yield Pareto optimality solutions might be discarded without solving the CLS problem. If $w^{0}=\hat{w}\left(\alpha^{0}\right)$ is a Pareto optimal vector, satisfying the conditions of Theorem 1 , then $\alpha^{0}$ is a proper scalar. The set of all proper scalars can be denoted by $\mathrm{B}, \mathrm{B} \subseteq \mathrm{A}$. Then $P=\{\hat{w}(\alpha) \mid \alpha \in \mathrm{B}\}$ represents the set of all Pareto optimal solutions, obtained from the set $L$ of all $\alpha$ optimal solutions to the CLS problem.

Firstly, we have to determine the boundary values $\alpha_{\min }$ and $\alpha_{\max }$ of the set of proper scalars B. Consider the single-objective optimisation problem (6). Its solution $w^{*}$ corresponds to the absolute minimum of the Total deviation $T^{*}=T\left(w^{*}\right)$, which cannot be improved by any other feasible vector $w \in Q$. The value of the second criterion, corresponding to this optimal solution gives the upper limit of $B$, $\alpha_{\max }=V\left(w^{*}\right)$. Evidently, if there are feasible priority vectors $w \in Q$ such that $V(w)=\alpha>\alpha_{\max }$, they cannot be Pareto optimal, since $T(w)>T^{*}$ and therefore there is no need to take them into consideration.

On the other hand, if (6) has multiple solutions $w_{i}^{*}, \mathrm{i}=1,2, \ldots, \mathrm{k}$, such that $T^{*}=T\left(\hat{w}_{i}\right)$, but resulting in different $V\left(\hat{w}_{i}\right)$, then $\alpha_{\max }=\min \left(V\left(\hat{w}_{i}\right)\right)$, since for all $\alpha>\alpha_{\max }$ the optimal vectors $\left(\alpha, T^{*}\right)$ do not satisfy (11), so the solutions are not Pareto optimal.

The lower bound $\alpha_{\min }$ of $\mathrm{B}$ can be obtained by solving the single optimisation problem (7). Generally, this problem has infinite number of solutions $w$, which lie on the line $V(w)=\alpha_{\min }$, but only the minimum value of the objective function $V^{*}$ is of interest. If the judgements are totally or strongly transitive, then $\alpha_{\min }=\alpha_{\max }=0$. In this case we have to solve only one CLS problem, although some of subsets $Q$ could be non-empty.

\subsection{Computational algorithm}

The computational procedure for solving the two-objective prioritisation problem can be summarised in the following algorithm.

Step 1: Solve the problem (6) and find $\alpha_{\max }$. If $\alpha_{\max }=0$, then set $\hat{w}=w^{*}$ and go to Step (6). Otherwise go to Step (2).

Step 2: Solve the problem (7) and find $\alpha_{\min }$. If $\alpha_{\min }=\alpha_{\max }$, then set $\hat{w}=w^{*}$ and go to Step (6). Otherwise go to Step (3).

Step 3: Form a set $\widehat{B}=\left(\alpha_{\text {min }}, \alpha_{\text {min }}+1 / 2, \alpha_{\text {min }}+1, \ldots, \alpha_{\text {max }}\right)$. 
Step 4: For each $\alpha \in \widehat{B}$ solve the CLS problem (10) and determine $\hat{w}(\alpha)$ and $\psi(\alpha)=T(\hat{w}(\alpha))$.

Step 5: Determine the set of the proper scalars $\mathrm{B} \subseteq \widehat{\mathrm{B}}$ and the entire set of Pareto optimal solutions $P=\{\hat{w}(\alpha) \mid \alpha \in \mathrm{B}\}$, using condition (11).

Step 6: Stop.

If the prioritisation problem is inconsistent and there are many Pareto optimal solutions, the decisionmaker should finally select a priority vector from the set $P=\{\hat{w}(\alpha) \mid \alpha \in \mathrm{B}\}$, best satisfying his/her preferences with respect to the accuracy and rank ordering.

\section{Example}

Let us consider a prioritisation problem with 5 unknown priorities. The comparison judgements provided by an expert are:

$a_{12}=5, a_{13}=1 / 3, a_{14}=7, a_{15}=2 / 3$;

$a_{23}=2, a_{24}=1 / 2, a_{25}=4$;

$a_{34}=1 / 2, a_{35}=1 / 4$

$a_{45}=1 / 3$.

From the first two steps of the algorithm we can find the ideal point $y^{*}=\left(T^{*}, V^{*}\right)$, shown in Fig. 1, and the boundary values $\alpha_{\min }=2, \alpha_{\max }=6$. The set of all candidates for being proper scalars is $\hat{\mathrm{B}}=(2,2.5,3, \ldots .5 .5,6)$.

Solving the CLS problem (10) for each $\alpha \in \widehat{B}$ and applying the optimality condition (11) we can determine the set of all Pareto optimal solutions. In this example all scalars in $\widehat{B}$ are proper, thus we have nine Pareto optimal solutions, given in Table 1.

Table 1. TOP solutions to the problem

\begin{tabular}{|c|c|c|c|c|c|c|}
\hline$w_{1}$ & $w_{2}$ & $w_{3}$ & $w_{4}$ & $w_{5}$ & $\mathrm{~T}$ & $\mathrm{~V}$ \\
\hline 0.283 & 0.124 & 0.123 & 0.186 & 0.284 & 55.261 & 2.0 \\
\hline 0.281 & 0.128 & 0.127 & 0.184 & 0.280 & 55.244 & 2.5 \\
\hline 0.390 & 0.129 & 0.128 & 0.130 & 0.224 & 41.716 & 3.0 \\
\hline 0.392 & 0.128 & 0.128 & 0.129 & 0.224 & 41.437 & 3.5 \\
\hline 0.358 & 0.103 & 0.102 & 0.060 & 0.376 & 31.373 & 4.0 \\
\hline 0.358 & 0.103 & 0.104 & 0.060 & 0.376 & 31.072 & 4.5 \\
\hline 0.361 & 0.110 & 0.110 & 0.059 & 0.361 & 30.942 & 5.0 \\
\hline 0.361 & 0.074 & 0.144 & 0.059 & 0.361 & 26.622 & 5.5 \\
\hline 0.566 & 0.117 & 0.224 & 0.093 & 0.421 & 26.479 & 6.0 \\
\hline
\end{tabular}

Fig. 1 shows graphically the Pareto optimal front $C$ as a number of points, represented by diamond symbols. From the entire set of non-dominated solutions the decision maker can select one solution, which best satisfies his preferences. For example, an appropriate compromise between accuracy and number of violations can be achieved by the solutions, corresponding to $\alpha=3$ and $\alpha=4$. 


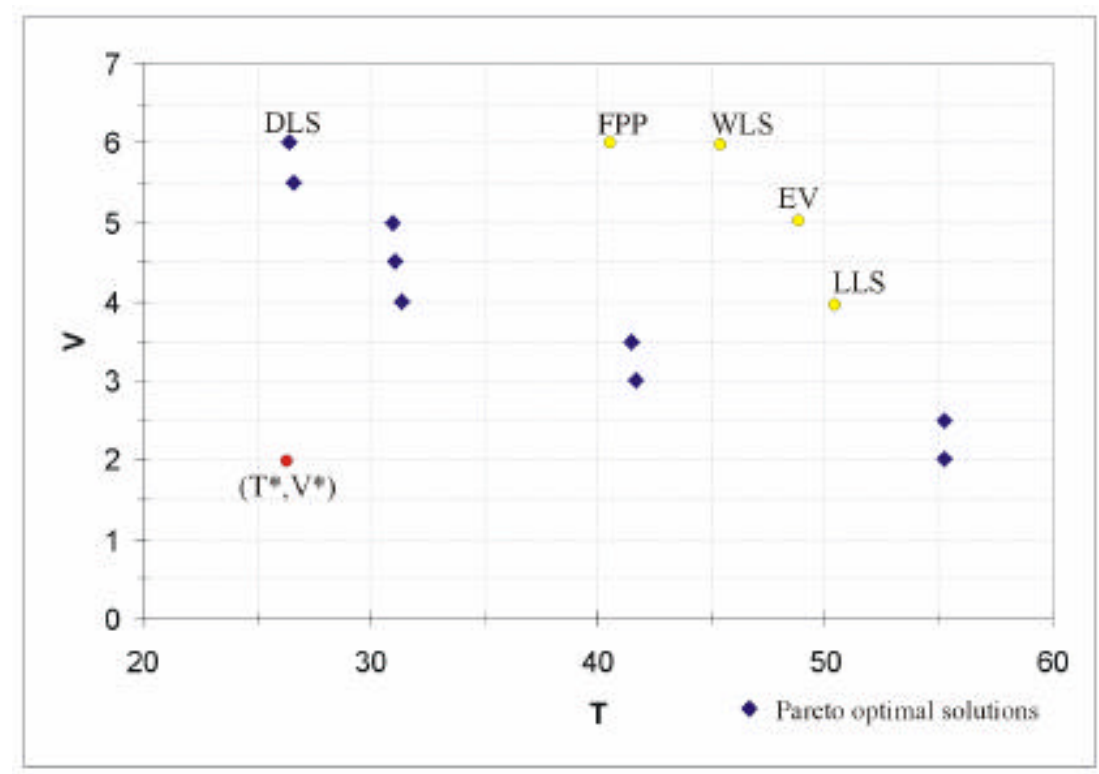

Fig. 1. Pareto optimal solutions

Solutions to this example by some other prioritisation methods are given in Table 2. They are also compared graphically in the objective space to the solutions, obtained by the proposed multiple-criteria optimisation method. As it can be observed from Fig. 1, the TOP solutions dominate strongly solutions, determined by all other prioritisation methods.

Table 2. Results from different prioritisation methods

\begin{tabular}{|c|c|c|c|c|c|c|c|}
\hline Method & $w_{1}$ & $w_{2}$ & $w_{3}$ & $w_{4}$ & $w_{5}$ & $\mathrm{~T}$ & $\mathrm{~V}$ \\
\hline DLS & 0.566 & 0.117 & 0.224 & 0.093 & 0.421 & 26.479 & 6 \\
\hline FPP & 0.401 & 0.145 & 0.234 & 0.103 & 0.117 & 41.079 & 6 \\
\hline WLS & 0.315 & 0.087 & 0.113 & 0.053 & 0.059 & 45.986 & 6 \\
\hline EV & 0.298 & 0.195 & 0.163 & 0.122 & 0.222 & 48.099 & 5 \\
\hline LLS & 0.287 & 0.182 & 0.136 & 0.137 & 0.257 & 51.416 & 4 \\
\hline GP & 0.670 & 0.134 & 0.067 & 0.096 & 0.033 & 477.504 & 5 \\
\hline
\end{tabular}

\section{Conclusions}

A new multiple objective prioritisation method is proposed in this paper, which minimises the Euclidean norm and the Number of rank violations, thus ensuring satisfactory accuracy and good rank preservation properties of the final set of Pareto optimal solutions. Comparisons to other prioritisation methods clearly show the advantages of this new approach to prioritisation in the AHP.

\section{References}

[1] Ali I., Cook W. and Kress M. (1986), "On the minimum violations ranking of a tournament", Management science, 32, 660-672.

[2] Barzilai J. (1997), “Deriving weights from pairwise comparison matrices”, Journal of Operational Research Society, 48, 1226-1232. 
[3] Blankmeyer E. (1987), "Approaches to consistency adjustments", Journal of Optimisation Theory and Applications, 54, 479-488.

[4] Bryson N., (1995), "A goal programming method for generating priority vectors", Journal of Operational Research Society, 46, 641-648.

[5] Chu A., Kalaba R. and Springarn K. (1979), "A comparison of two methods for determining the weights of belonging to fuzzy sets", Journal of Optimisation Theory and Applications, 27, 531-541.

[6] Cook W. and Kress M. (1988), "Deriving weights from pairwise comparison ratio matrices: An axiomatic approach", European Journal of Operational Research, 37, 355-362.

[7] Crawford G. and Williams C. (1985), "A note on the analysis of subjective judgement matrices", Journal of Mathematical Psychology, 29, 387-405.

[8] Despotis D. (1996), "Fractional Minmax goal programming: A unified approach to priority estimation and preference analysis in MCDM", Journal of Operational Research Societ, 47, 989-999.

[9] Fichtner J. (1986), "On deriving priority vectors from matrices on pairwise comparisons", Socioeconomic Planning Science, 20(6), 341-345.

[10] Golany B. and Kress M. (1993), "A multicriteria evaluation of methods for obtaining weights from ratio-scale matrices", European Journal of Operational Research 69, 210-220.

[11] Jensen R. (1984), "An alternative scalling method for priorities in hierarchical structures", Journal of Mathematical Psychology 28, 317-332.

[12] Lin J. (1976), "Multiple-objective problems: Pareto-optimal solutions by method of proper equality constraints", IEEE Transactions on Automatic Control 21 (5), 641-650.

[13] Lin J. (1976), "Three methods for determining Pareto-optimal solutions of multiple-objective problems". In: Y. Ho, S. Miller (Eds.), Directions in large-scale systems. Many-person optimisation and decentralised control, Plenum Press, NY, 117-139.

[14] Mikhailov L. (2000), "A fuzzy programming method for deriving priorities in the analytic hierarchy process", Journal of Operational Research Society, 51, 341-349.

[15] Mikhailov L. and Singh M. (1999), "Comparison Analysis of Methods for Deriving Priorities in the Analytic Hierarchy Process", Proceedings of IEEE Conference on Systems, Man and Cybernetics, Tokio, Japan, 1037-1042.

[16] Saaty T. and Vargas L. (1984), "Inconsistency and rank preservation", Journal of Mathematical Psychology 28, 205-214.

[17] Saaty T. and Vargas L. (1984), "Comparison of eigenvalue, logarithmic least squares and least squares methods in estimation ratios", Mathematical Modelling 5, 309-324.

[18] Saaty T. (1977), "A scaling method for priorities in hierarchical structures", Journal of Mathematical Psychology 15, 234-281.

[19] Saaty T. (1990), Eigenvector and logarithmic least squares, European Journal of Operational Research 48, 156-160.

[20] Sawaragi Y., Nakyama H., and Tanino T. (1985), Theory of multiobjective optimisation, Academic Press, Orlando.

[21] Szidarovsky F, Gershon M., and Duckstein L. (1986), Techniques for multiobjective decision making in systems management, Elsevier, Amsterdam.

[22] Takeda E., Cogger K. and Yu P. (1987), "Estimating criterion weights using eigenvectors: A comparative study", European Journal of Operational Research 29, 360-369.

[23] Kumar N. and Ganesh L. (1996), "A simulation-based evaluation of the approximate and the exact eigenvector methods employed in AHP”, European Journal of Operational Research, 95, 656-662. 